\title{
Ocular Prosthesis: Indications to Management
}

\author{
Malinda Cafiero-Chin, OD, \\ Cathy Marques, OD, FAAO, \\ Heather J. Danz, BCO, BADO
}

385 Tremont Ave.

East Orange, NJ 07018

973-676-1000 x 3917

Correspondence may be directed to malinda.cafiero@va.gov

\section{Abstract}

Caring for patients with a prosthetic eye can be a challenge to clinicians. Regardless of the circumstances leading to eye removal, inspection of the underlying tissue should be part of a comprehensive eye exam. Maintaining the overall health of the anophthalmic socket is critical in patient comfort and optimal prosthetic fit. Discussions will focus on anophthalmic procedures and preparation of the socket for prosthetic fitting. Care and management of the prosthesis and the anophthalmic socket, including associated ocular tissue disorders will be emphasized. The article will enhance the clinician's comfort level managing patients wearing ocular prosthesis.

KEY WORDS: ocular prosthesis, enucleation, evisceration, exenteration, orbital implants

Résumé

Le soin des patients qui ont une prothèse oculaire peut poser un défi aux cliniciens. Quelles que soient les circonstances à l'origine de l'ablation de l'œil, un examen complet de la vue doit inclure l'inspection des tissus sousjacents

Il est crucial de maintenir la santé générale de la cavité orbitaire anophtalmique pour le confort du patient et l'ajustement optimal de la prothèse.

Les discussions porteront sur les interventions anophtalmiques et la préparation de la cavité orbitaire pour l'ajustement de la prothèse. On mettra l'accent sur le soin et la prise en charge de la prothèse et de la cavité orbitaire anophtalmique, y compris les troubles des tissus oculaires connexes. L'article permettra aux cliniciens qui traitent des patients portant une prothèse oculaire de se sentir plus à l'aise. 


\section{BACKGROUND}

The need for an ocular prosthetic device knows no boundaries. The population that can be affected by ocular disease or trauma is not dictated by age, sex or race. Regardless of the mode of practice, the clinician will likely see patients who have had an anophthalmic procedure, the vast majority of whom will be wearing an ocular prosthesis. Because complications may arise with either the prosthetic eye wear or the anophthalmic socket, it is important for the eyecare provider to have a firm understanding of management of these cases, as well as when to make appropriate referrals to the ocularist and the ophthalmic surgeon.

\section{SURGICAL PROCEDURES}

To understand the complications associated with prosthetic wear, one must first understand anophthalmic procedures. The three main surgical techniques used in the partial or complete removal of the eye are evisceration and enucleation, respectively, which are the most common, and exenteration which is the least common. Having a grasp of the indications, procedures and outcomes involved with each of these surgeries helps to educate patients and to manage expectations prior to their meeting with the surgeon.

Enucleation involves the complete removal of the globe, along with a portion of the optic nerve, while maintaining the surrounding orbital tissue. This provides the surgeon with a large, intact histological specimen for review, which is particularly important for patients with a known intraocular malignancy in which non-surgical treatment options have either failed or are not an option and in which the eye cannot be preserved. ${ }^{1}$ Intraocular tumors have become the primary indication for enucleation and would include, but are not limited to, retinoblastomas and uveal melanomas. ${ }^{1,2,3,4,5,6}$ For patients presenting with phthisical eyes of unknown etiology but which require removal of the eye, enucleation would once again be the procedure of choice. ${ }^{1}$ In these cases, it is assumed an underlying malignancy may have caused the degeneration, requiring entire removal of the globe. Other indications for enucleation include severely traumatized eyes ${ }^{5}$ which may be at risk for sympathetic ophthalmia. Early enucleation is a point of contention amongst eye care providers because of the infrequent incidence of sympathetic ophthalmia, ranging from $0.001 \%$ to $2.0 \%$ of traumatized eyes ${ }^{5}$, as well as the uveitis management options available. ${ }^{5,7,8,910}$ While traditionally enucleation would be the anophthalmic procedure of choice in sympathetic ophthalmia, some studies are finding that evisceration may also be a viable option. ${ }^{8,9}$ Patients with chronic pain due to end stage neovascular glaucoma or chronic uveitis may undergo either enucleation or evisceration.

On an outpatient or inpatient basis, the enucleation procedure is usually performed under general anesthesia, but can be performed under local anesthesia with a retrobulbar block alone in warranted cases. ${ }^{5,11}$ After the conjunctiva and Tenon's capsule are dissected, the extra ocular muscles are tagged with a stitch and transected from the globe, and the optic nerve is severed, allowing for removal of the intact globe. To increase the chance of complete removal of the known or suspected cancerous tissues, a large portion of the optic nerve is usually removed. ${ }^{5}$ There is a risk of ptosis and extra ocular muscle damage during enucleation because the optic nerve is transected near the orbital apex.

Due to so much of the natural orbital volume being lost, an orbital implant is then introduced into the remaining space via a transducer. The extra ocular muscles are reattached to the orbital implant and Tenon's capsule and the conjunctiva sutured. An antibiotic ointment is instilled and a conformer, which helps maintain the conjunctival space, is then placed over the tissue. The area is then pressure patched for 24-48 hours.,11 Appropriate analgesics are prescribed for patients, who typically will encounter some pain and headache for the first few days. Once the pressure patch is removed, an antibiotic or antibiotic/steroid combination ointment is usually applied twice a day for one week.,11 At one week post-surgery, the conformer is briefly removed for inspection of the remaining ocular tissue for signs of infection and for assurance of proper healing.

Evisceration differs from enucleation in that it involves the surgical removal of the contents within the globe, while preserving the sclera, extraocular muscles and optic nerve. Indications for evisceration typically include blind, painful eyes found in such cases as chronic uveitis or 
neovascular glaucoma, as well as those with corneal perforation. Important indications with evisceration are patients with endophthalmitis requiring anophthalmic surgery. There is decreased risk of spreading the infectious material through the dura into the intracranial spaces since so much of the orbital tissue remains intact during this procedure. Currently there is debate on whether enucleation can also be performed in these situations, particularly since the literature suggests an increased risk of sympathetic ophthalmia following evisceration. ${ }^{12}$ However, evisceration continues to be the procedure of choice among surgeons in the majority of these cases. $^{6}$

The evisceration procedure involves an incision near the limbus, with the cornea either removed or flapped, after which the surgeon removes the inner contents of the eye through the use of an evisceration spoon. The internal aspect of the sclera is cauterized and treated with absolute alcohol to denature any residual proteins, decreasing the risk of sympathetic ophthalmia. As with enucleation, the lost orbital volume is replaced with an orbital implant and the sclera and conjunctiva are then sutured. Unlike enucleation, there is no need for extra ocular muscle reattachment because they were left undisturbed. An antibiotic ointment and a conformer are then placed over the area and the eye is pressure patched, with much of the post-operative care mimicking that of enucleation.

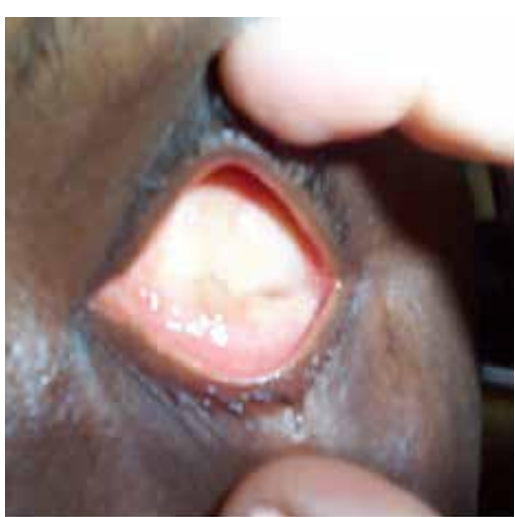

Figure 1. Healthy anophthalmic socket
The short term goals after both enucleation and evisceration are deep fornices to hold the conformer and eventually the prosthesis, a socket lined with healthy conjunctival membranes, and a normal appearance of the lid. (Figure 1) The aforementioned conformer plays an important role in the eventual successful fit of the prosthesis by not only helping to stabilize the implant during the healing process, but by also reducing the risk of tissue contracture of an anophthalmic socket. Typically the conformer is made of either acrylic or silicone and is left in the conjunctival fornices for 4-6 weeks ${ }^{5}$ helping to maintain the space that will eventually accommodate the prosthesis.

The eventual cosmetic success of the overlying prosthesis is also largely due to the orbital implant introduced into the socket during enucleation and evisceration. During surgery, the surgeon can use a sizing sphere to determine the appropriate size; typically a $20 \mathrm{~mm}$ sphere for adults. ${ }^{11,13} \mathrm{The}$ appropriate sized sphere helps create tension free closure of Tenon's capsule and conjunctiva, ${ }^{11}$ minimizing complications post operatively. In addition to replacing the lost volume occurring during surgery, an orbital implant maintains the orbital structure and reduces scar formation. The implant either directly or indirectly helps to impart movement to the overlying prosthesis, as is the case with enucleation and evisceration respectively, or therefore contributes to the ultimate goal of having a prosthetic eye that looks and moves similarly to the contralateral eye.

Exenteration is a procedure involving removal all of the tissues within the socket and the entire orbit, typically including the conjunctiva, globe, orbital fat, part or all of the lids and sometimes a portion of the bony orbit. This most radical of procedures is reserved for patients with large, highly invasive orbital tumors which may have originated from within the orbit or from the surrounding orbital structures. ${ }^{14}$ These tumors can be highly destructive, and may have the capacity to extend into the intracranial spaces, proving eventually fatal for patients. Many of these patients have a history of malignant tumors of the eye lids, often squamous cell carcinomas and basal cell carcinomas ${ }^{15,1,6,1,118}$ as well as other highly invasive tumors of the conjunctiva, intraocular structures and orbit ${ }^{14}$ including intraocular malignant melanomas that have extended outside of the globe and which may not have responded to non-surgical treatment options such as radiation and chemotherapy.,19 
Patients who have undergone exenteration often require long term use of a combination antibiotic and steroid ointment, as well as long term analgesics for management of chronic orbital pain. Depending on the extent of tissue and bony areas removed, there may be longterm complications of sinusitis, ear problems, orbital cyst ingrowth, infection and malignancy recurrence. There is also the strong possibility of psychosocial issues that go along with losing such a large, obvious portion of the face. Some patients will decide to use a facial prosthesis but many find the facial adhesive used to be highly irritating and often discontinue use. There have been some strides made with the use of titanium osseo integrated implants to which the prosthesis is attached by magnets or clips. ${ }^{15}$ Another alternative that patients may opt for is the spectacle mounted facial prosthesis. (Figure 2) Overall the use of a facial prosthesis is limited, with most patients preferring to patch the area ${ }^{15}$ and also because of the mortality rate associated with recurrence of the malignancy.

\section{ORBITAL IMPLANTS}

Orbital implants are typically made of either non-porous or porous materials. (Figure 3) Nonporous alloplastic implants can be comprised of varied inert materials, including the more commonly used silicone and polymethyl methacrylate (PMMA). ${ }^{6}$ These materials are made into solid, spherical implants that are well tolerated, have a low extrusion risk and are a good choice for implants that will not be integrated, or pegged. These materials are especially important for patients who have had infections in which porous implants are no longer an option. To decrease the risk of implant migration and to improve motility, the implant can be wrapped in a material such as donor sclera, providing the option of direct extra ocular muscle attachments, depending on the surgery performed.

Porous materials, also known as biocompatible or biointegrated materials are now more commonly used as the initial implant material with porous polyethylene, hydroxyapatite and aluminum oxide being the most common. ${ }^{6,11,20,21}$ Due to the porous nature of these materials, fibrovascular ingrowth occurs, allowing for improved implant stability as well as decreasing rejection risk. Porous polyethylene is a smoother, softer material to which the extra ocular muscles can be directly attached during enucleation. ${ }^{5,13}$ In contrast, hydroxyapatite creates a rough, abrasive surface. To protect the overlying

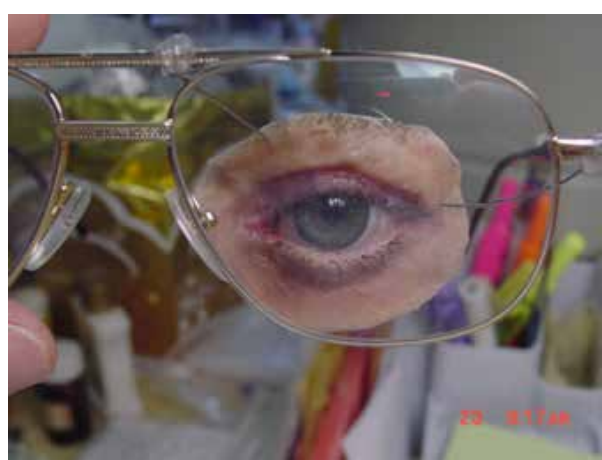

Figure 2. Spectacle mounted prosthesis.
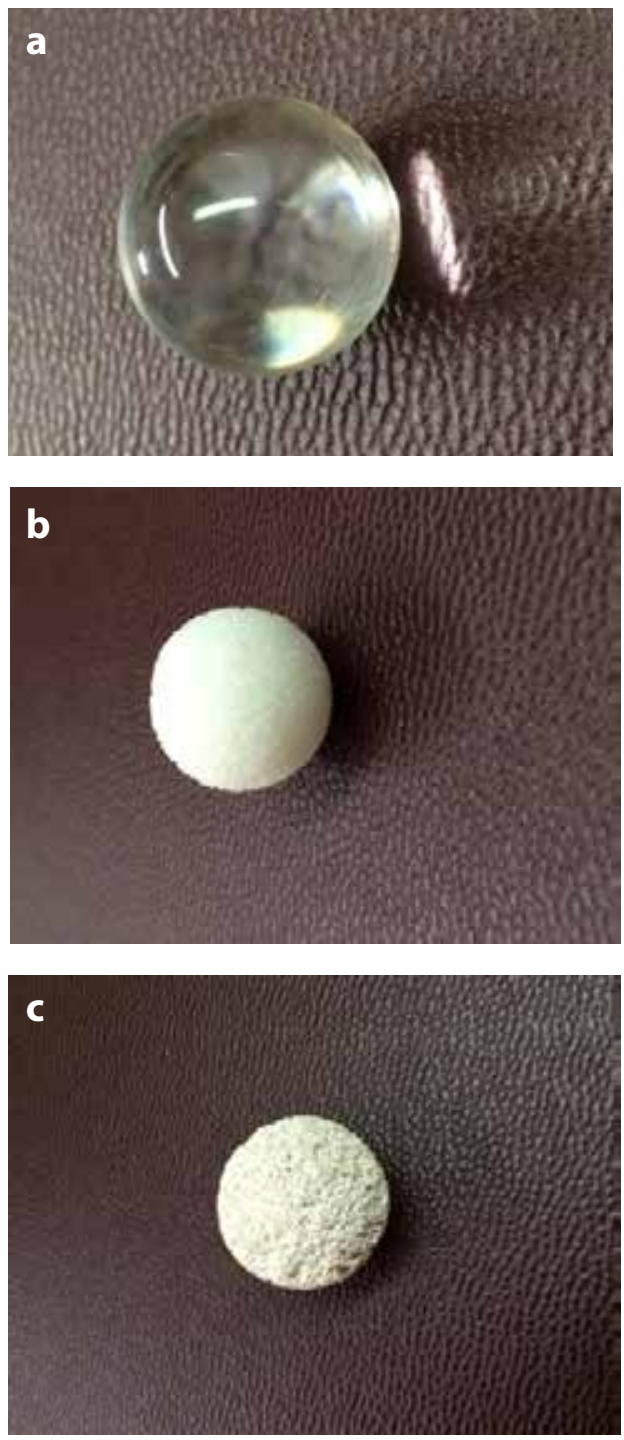

Figure 3. Various orbital implant materials. a) polymethyl methacrylate (PMMA), b) porous polyethylene), C) hydroxyapatite 


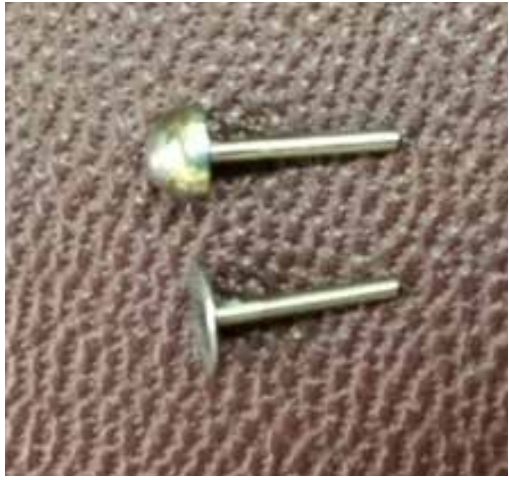

Figure 4.Peg

tissue and to serve as a scaffold for reattachment of the extraocular muscles, the hydroxyapatite implant is wrapped in materials ranging from donor sclera or autologous tissue to synthetic vicryl or polyglactin mesh., ${ }^{5,21}$ Aluminum oxide as an implant material is gaining popularity due to its relative lower cost, as well as suggested excellent biocompatibility. ${ }^{21}$

The use of a porous orbital implant allows for the optional integration of the implant with the overlying prosthesis by incorporating a peg. (Figure 4) This involves an additional procedure performed typically 6 months or more after the initial anophthlamic surgery. ${ }^{5,21}$ A fairly quick procedure done under local anesthesia, the surgeon will open the conjunctiva and use a specialized tool to bore a hole into the implant. A sleeve is then placed into the opening to serve as a docking station for the peg to sit. Afterwards, the ocularist will modify the posterior portion of the patient's prosthesis. The goal is to have the peg sit flush with the implant offering the possibility of a better range of movement of the overlying prosthesis. Pegging of the orbital implant also helps decrease the weight on the lower lid, decreasing the chance of lid sagging. Aside from having an additional surgical procedure, disadvantages include the risk of infection and chronic discharge from implant exposure, as well aberrant movement of the prosthesis secondary to improper peg placement. There are studies being conducted looking at using pegs from the initial onset of anophthalmic surgery, as well as exploring quasi-integrated orbital implant designs in which the posterior prosthesis combines with the anterior portion of the orbital implant in a "lock and key" fashion. ${ }^{21}$

\section{FABRICATION/CARE OF OCULAR PROSTHESIS}

After the wound has healed, approximately six to eight weeks after surgery, an ocular prosthesis can be fitted. ${ }^{22,23}$ A prosthetic device can be fabricated in two forms, a scleral shell or a

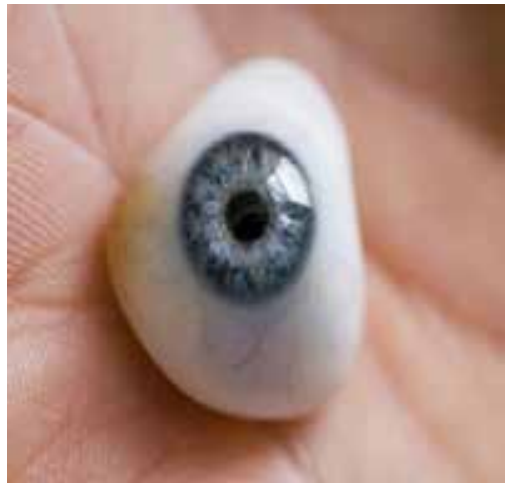

Figure 5. Ocular prosthesis made from acrylic

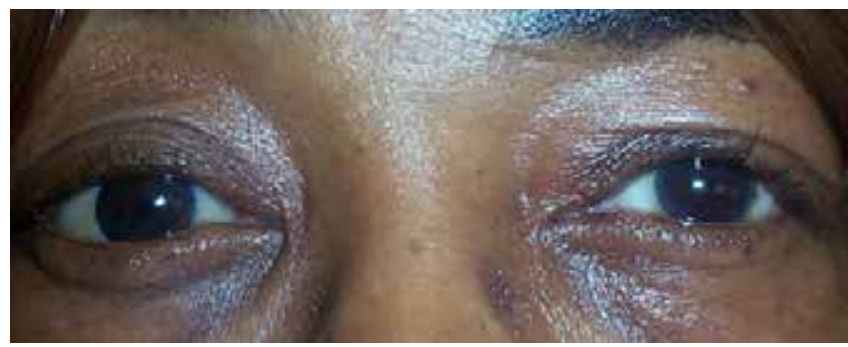

Figure 6. Ocular prosthesis: right eye. Note matching iris color and scleral hue. full thickness prosthesis. A scleral shell is fit over a phthisical eye and a full thickness prosthesis is fit over the anophthalmic socket. ${ }^{24}$ (Figure 5, 6) The patient should be reassured that this is a pain free process. The time to produce a custom fit prosthetic device will be dependent upon the number of modifications that need to be made. An average time is about one week.

Prosthetic devices can be fit either from a stock set of pre-fabricated eyes or can be custom made. Ideally, a custom made prosthetic eye is preferred to increase stability and aid in movement as the contour of the anophthalmic socket is taken into consideration..$^{25}$ There are various materials and methods used in the fabrication of a prosthetic device. The materials used today vary from the prosthetic device of the past. Glass, although still used in some areas of Europe, has given way to more durable materials, such as poly methyl-methacrylate which is chosen for its durability, biocompatibility, availability and cost. ${ }^{24,25}$ The fitting method chosen is at 
the discretion of the ocularist. One of the most common fitting techniques is the impression fitting.

The impression fitting method involves injecting alginate material directly into the patient's orbit using an impression tray. ${ }^{24,26}$ When the substance hardens, it is removed from the orbit and then can be adjusted to form the front surface of the device using wax. The iris and pupil are then positioned taking into account the appearance of the fellow eye. The mold is then filled with methyl-methacrylate resin that is liquid acrylic. Various instruments are used to eliminate the formation of air pockets within the plastic. The mold is then heat treated to harden the liquid. After this stage, the device is hand painted to reflect the unaffected eye. ${ }^{26}$ Although fabrication techniques vary, a prosthesis that is customized is preferred over stock eyes that have not been modified in any way. Improvement in comfort and cosmesis can be achieved as each anophthalmic socket is unique..$^{27}$ (Figures 7, 8, 9)

Regardless of the method used to create the prosthetic device, at each eye exam, the device should be removed from the patient's socket and inspected for defects. Removal of the prosthetic device is similar in fashion to the removal of a hard contact lens. A suction device, typically larger than that used with a hard contact lens, can be used to insert and remove the device easily. In the event that a suction device is unavailable, the practitioner, as well as the patient, should be comfortable with digital manipulation of the prosthesis. ${ }^{25}$ When using the fingers for removal, the prosthesis should be pushed up over the lower eyelid. This will allow the device to slide out from the bottom. Conversely, when inserting the device, the top should be inserted first by pushing up under the upper eyelid and sliding the lower part under the lower eyelid. The superior portion of the prosthesis is typically thicker and larger than the lower portion to aid in maintaining superior lid position and can also help with orientation during insertion. ${ }^{27}$

General eye care for the patient wearing a prosthetic device requires a detailed history of the patient's wearing habits and how often and in what manner the device is removed and cleaned. The practitioner should ask how old the current device is and how often he/she sees the ocularist. A general guide for prosthesis replacement is approximately every five years to accommodate for changes to the socket. ${ }^{23}$ It is recommended that patient should see the ocularist every six months for polishing and adjustments to the device at least annually. ${ }^{23,24}$

To ensure a comfortable fit, inspection of the prosthesis is important. The device should be smooth and free of sharp edges. If the device does not feel smooth, it can be washed with soap and water or gas permeable contact lens solution. If it continues to feel rough, then the device likely requires polishing, contouring or replacement. ${ }^{23}$ Polishing of the ocular prosthesis, unlike polishing of a gas permeable contact lens, cannot be done in office and requires a referral to the ocularist. Contouring to ensure proper fitting in the changing socket requires specialized equipment and also requires the ocularist's expertise. ${ }^{23}$ (Figures $10,11)$

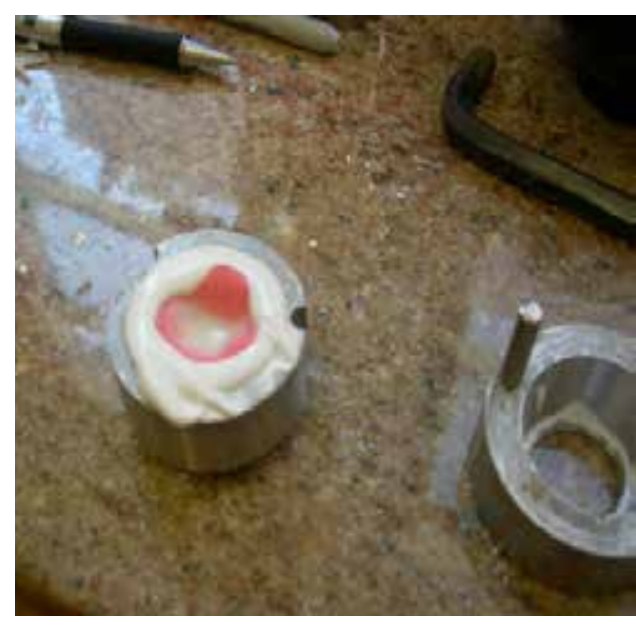

Figure 7. Cast used to make custom prosthesis.

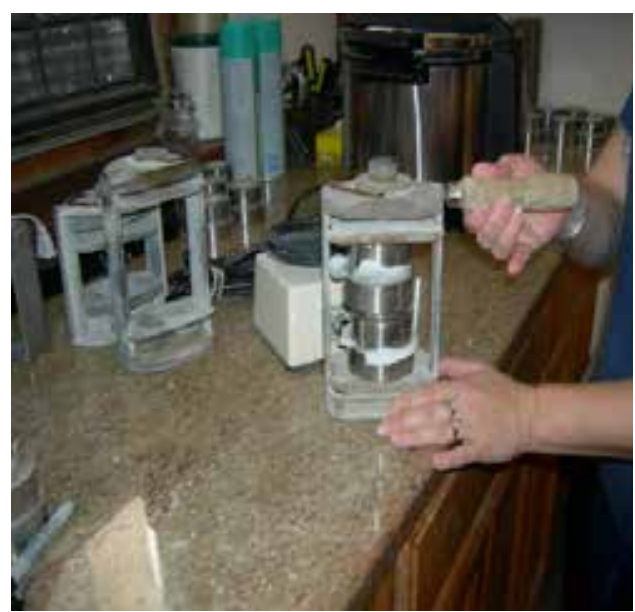

Figure 8. Use of Carver press to remove air from custom prosthesis prior to hardening.

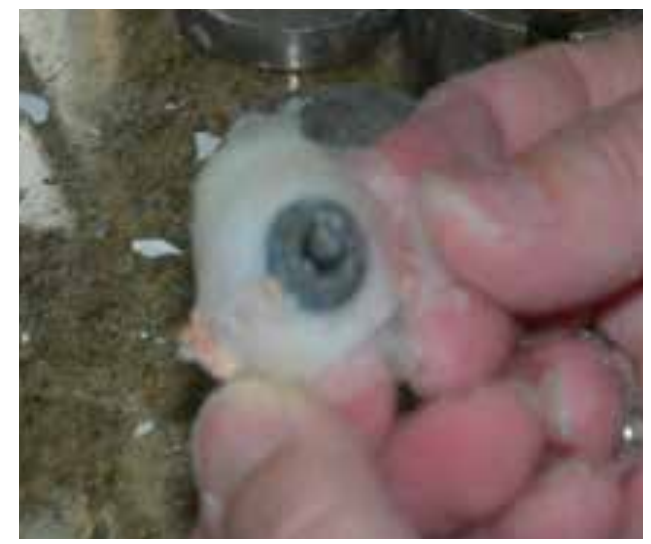

Figure 9. Custom prosthesis after curing process upon removal from mold. 


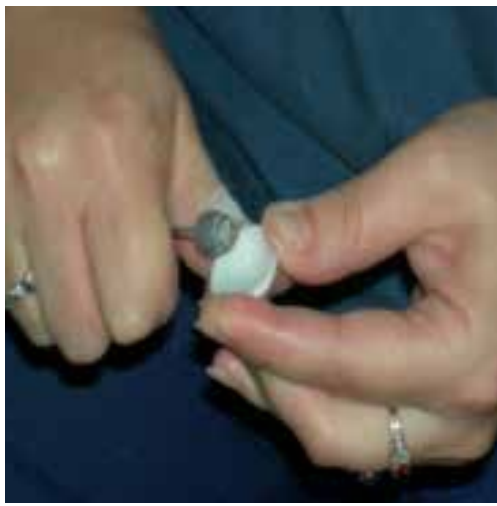

Figure 10. Modification of prosthetic device.

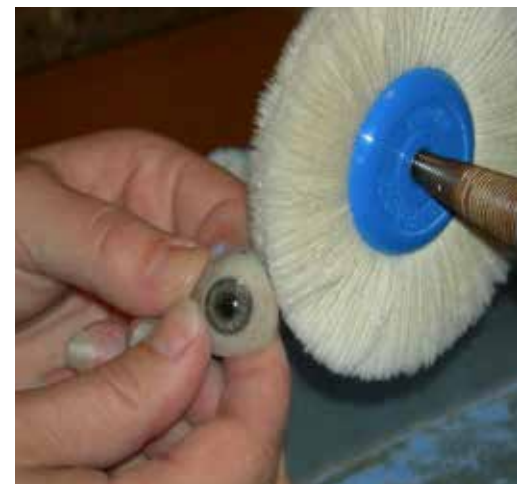

Figure 11. Polishing the prosthesis.
In addition to inspection of the prosthetic device, the patient should be questioned regarding care of the device. Because ocular prosthetics are custom made, care is custom as well. What may be standard for one patient could be completely different for another. The patient should limit the removal of the prosthesis. Removing the device too often can lead to increased mucous production and irritation of the orbital tissue. The device should be left in place and only removed for cleaning approximately once per month. ${ }^{22,23}$ The recommendation for cleaning the prosthesis will be dependent upon the mucous and protein deposition. The prosthesis can be cleaned with soap and water, baby shampoo and water, or gas permeable contact lens cleaner. ${ }^{23,24}$

\section{COMPLICATIONS}

In addition to inspecting the device, it is important for the eye care provider to inspect the ocular tissue for pathology. The most common ocular tissue disorders encountered include discharge, dry eye, discomfort, implant exposure, pain, ptosis, lid laxity, expulsion, adhesions and problems associated with the peg. ${ }^{28}$

\section{DISCHARGE}

Discharge can be caused by mechanical irritation or infection. In a study conducted by Pine, et al. 93\% of prosthetic eye wearers reported watering, discharge and crusting at some time during prosthesis wear with $60 \%$ reporting these symptoms daily. ${ }^{29}$ Just as would be done for a patient with a seeing eye, the color of the mucous is important. A white, stringy discharge typically represents mechanical irritation and/or allergy. Inspect the tissue for signs of giant papillary conjunctivitis. Alford recommends prednisolone acetate $1 \%$ three times a day for fourteen days for advanced cases and cromolyn sodium or topical NSAIDs for less severe cases. ${ }^{22}$ To reduce the incidence of recurrence, question the patient about his/her cleaning habits and recommend alternatives. Discuss the use of lubricant drops and determine the last time the device was professionally polished.

The presence of green or yellow discharge in conjunction with red orbital tissue with or without an odor can signify an infection. A course of topical antibiotics should be initiated while recalcitrant cases should be cultured. The fellow eye should always be examined with or without suspected infection. As with any infection or inflammation, treat appropriately with drops and/or ophthalmic ointments to combat the response and consider having the device polished.

\section{DRY EYE}

Dry eye is a common symptom among prosthesis wearers. Recommendations for treatment will be dependent on the severity of the patient's symptoms. A mild dry eye can be treated with viscous ocular lubricants three to four times a day. Just as with the sighted dry eye patient, a lubricant with longer lasting power may be needed. In that case, bland ophthalmic ointment or silicone oil should be used. Bland ophthalmic ointment can be placed in the upper or lower cul-de-sac. This is determined by patient preference or how shallow the inferior portion of the prosthesis fits in the lower orbital area. If the lower orbital area is shallow, inserting ointment in the upper eyelid area may insure the prosthesis does not become displaced by manipulation of the lower lid. Many patients find $100 \%$ medical grade silicone oil to be a comfortable lubricant. It lasts longer than the viscous artificial tears, yet is thinner than the ointment. Silicone oil can be easily purchased by the bottle or box online from various companies. 
A small sample study concluded that cyclosporine A $0.05 \%$, (Restasis, Allergan), has shown subjective improvement in ocular comfort in prosthetic wearers. ${ }^{30}$ This finding lends itself as a possible option for those with dry eye complaints in which conventional artificial tears were not successful. Larger cohort studies are necessary to determine both the long term benefit and cost consequence of using cyclosporine A drops in this population. This finding may be an additional benefit if blepharitis and meibomian gland dysfunction are found as these often contribute to dry eye. If after addressing the aforementioned dry eye and/or eyelid findings the patient continues to experience symptoms, a professional polish must always be considered.

\section{OCULAR DISCOMFORT}

Ocular discomfort or pain while wearing the prosthesis can lead to an inability in wearing the prosthesis successfully and requires the clinician to observe both the device and the orbital area. If there are no defects found in the hardware itself, the orbital tissue in and around the socket must be scrutinized; several reasons must be considered including malignancy, systemic disease, contracted socket syndrome and implant exposure. In these cases, a thorough history including why the patient lost the eye, details about the ophthalmic surgical history, overall systemic health, and wear schedule for the device can aid the clinician in making the proper diagnosis.

If anophthalmia was from a malignancy, then tumor growth or metastasis posterior to the implant must be considered. Systemic disease such as thyroid eye disease may cause the prosthesis to not fit into the orbit properly. Since the patient's extraocular muscles are typically preserved, the implant may be pushed anteriorly causing a shallow orbit and an ill-fitting prosthesis. In these instances, a referral to the patient's primary care physician or oncologist is in order.

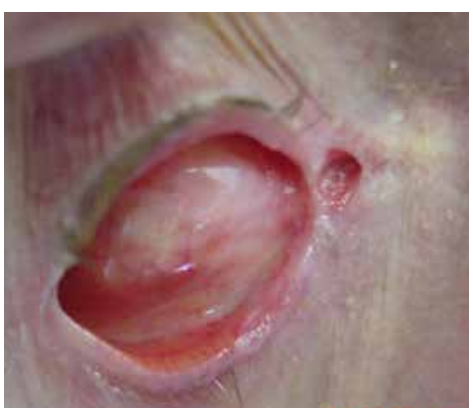

Figure 12. Adhesion of puncta.

Contracted socket syndrome can be another cause of an improper fit. This syndrome causes the conjunctiva to tighten as a result of inflammation and fibrosis. ${ }^{31}$ This can occur if a patient is not wearing the prosthesis as directed or as a result of direct trauma, surgical insult, burns, cicatricial disease or irradiation. ${ }^{23}$ Surgical intervention is required in these cases. Typically mucous membrane grafting is the treatment of choice; however recent studies suggest the use of amniotic membranes to reconstruct the orbit, postauricular full thickness skin grafts or microvascular radial forearm free flaps with successful repair. ${ }^{32,33,34}$ In addition to contracture, adhesions can form within the tissue of a patient not wearing the prosthesis properly. Tissue such as the eyelids, conjunctiva and puncta may adhere to each other making the area unable to hold the prosthesis in place properly and also causing expulsion. Surgical correction of the adhesion will allow for proper refitting of the prosthetic device. (Figure 12)

If pain ensues due to implant exposure, measure the size of the defect. Exposure can occur at any time post-anophthalmic surgery and must be looked for at each visit. It has been noted with porous implants, exposure between three and twelve months post-op can be due to poor surgical technique, infection, inflammation, inadequate tissue healing or erosion of tissue over the implant or pressure from the prosthesis. Exposure after two years was most likely from pressure from the prosthetic device and friction from both mechanical and inflammatory sources. ${ }^{13}$ Jordan also noted a $2 \mathrm{~mm}$ defect found four weeks after surgery that closed spontaneously over a two week period. However, if the tissue surrounding the exposure appears infected or infection is a concern, antibiotics drops may be used. Additionally, alterations in the prosthesis should be considered to allow for an improved fit over the affected area. Defects persisting beyond six to eight weeks require a consultation with the surgeon as grafting may be necessary to close the wound. ${ }^{29}$

\section{LID COMPLICATIONS}

Ptosis and lid laxity are common problems encountered by this population as well. Ptosis can be alleviated by lifting the superior edge of the prosthesis allowing it to fit under the levator to improve lid position. If this has been done several times or if the prosthesis is not fit properly or 
worn in the appropriate manner, lifting the edge will no longer lift the lid. In these cases, referral to the surgeon should be made for repair. If the ptosis is suspected to be related to the contracted socket, repair of the orbital volume may correct the ptosis in $30 \%$ of cases according to Jordan. ${ }^{28}$ If the ptosis is due to lid laxity, a study conducted by Mombaerts and Groest, ${ }^{35}$ suggests that a preparatory prosthesis can be used to align the pupils prior to ptosis surgery to alleviate moderate to severe ptosis.

\section{PEGGED IMPLANTS}

There are some problems intrinsic to those fitted with a peg. Examining the tissue surrounding the peg and the peg itself is extremely important in these patients. It has been estimated that patients receiving a free standing polycarbonate peg had a complication rate of approximately $71 \%{ }^{36}$ Notable

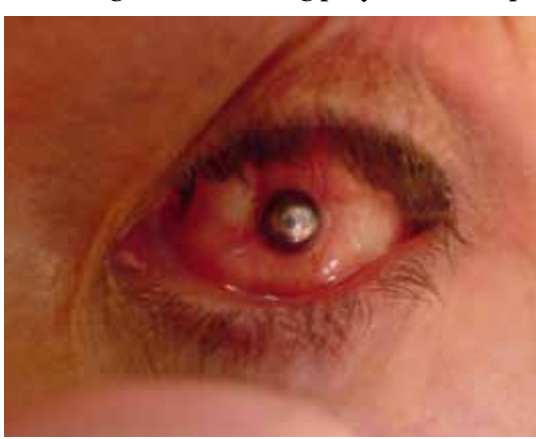

Figure 13. Inflammation caused by peg complication. complications include: pyogenic granuloma at peg site, loose pegs, conjunctival overgrowth and peg extrusion. If the peg is broken, it will not align properly with the posterior surface of the prosthesis and will not stay in place or move properly. Conjunctival tissue may begin to grow over the peg causing interference with the flush posterior surface of the prosthesis and would need to be corrected surgically. Similarly, the conjunctiva may also regress around the peg causing exposure near the sleeve making the peg loose and increasing the patient's likelihood of infection. Any of these processes would need referral for surgical evaluation. (Figure 13)

\section{CONCLUSION}

Overall working with anophthalmic patients is rewarding when the clinician is comfortable looking for the signs of complications due to underlying ocular tissue or socket disorders and device irregularities. To provide the patient with the most comprehensive eye care, the most important procedure is removal of the device for inspection of the tissue and prosthesis, treat the underlying tissue disorders and/or refer to the appropriate specialist be it the ocularist or the surgeon.

\section{REFERENCES}

1. The Anophthalmic Socket. In: Holds, JB, et al, eds. Orbit, Eyelids, and Lacrimal System, Section seven. Basic and Clinical Science Course. Singapore: American Academy of Ophthalmology, 2010: 123-132.

2. Sengupta S, Krishnakumar S, Biswas J, et al. Fifteenyear trends in indications for enucleation from a tertiary care center in South India. Indian J Ophthalmol 2012; 60(3):179-82.

3. Setlur VJ, Parikh JG, Rao NA. Changing causes of enucleation over the past 60 years. Graefes Arch Clin Exp Ophthalmol 2010; 248(4):593-7.

4. Collaborative Ocular Melanoma Study Group. Trends in size and treatment of recently diagnosed choroidal melanoma, 1987-1997: findings from patients examined at collaborative ocular melanoma study (COMS) centers: COMS report no. 20. Arch Ophthalmol 2003; 121(8):1156-1162.

5. Moshfeghi DM, Moshfeghi AA, Finger PT. Enucleation. Surv Ophthalmol 2000; 44(4):277-301.

6. Shah RD, Singa RM, Aakalu VK, Setabutr P. Evisceration and Enucleation: A National Survey of Practice Patterns in the United States. Ophthalmic Surgery, Lasers and Imaging 2012: 425-30.

7. Damico FM, Kiss S, Young LH. Sympathetic ophthalmia. Semin Ophthalmol 2005 Jul-Sep;20(3):191-7.

8. Gürdal C, Erdener U, Irkeç M, Orhan M. Incidence of sympathetic ophthalmia after penetrating eye injury and choice of treatment. Ocul Immunol Inflamm 2002 Sep; 10(3): 223-7.

9. du Toit N, Motala MI, Richards J, Murray AD, Maitra S. The risk of sympathetic ophthalmia following evisceration for penetrating eye injuries at Groote Schuur Hospital. Br J Ophthalmol 2008 Jan; 92(1): 61-3.
10. Chan CC, Roberge RG, Whitcup SM, Nussenblatt RB. 32 cases of sympathetic ophthalmia. A retrospective study at the National Eye Institute, Bethesda, Md., from 1982 to 1992. Arch Ophthalmol 1995 May; 113(5): 597-600.

11. Morton A. Enucleation and Evisceration. In: Thach, $\mathrm{AB}$ ed. Ophthalmic Care of the Combat Casualty (Textbooks of Military Medicine). Washington, DC: Office of The Surgeon General at TMM Publications, 2003: 405-420

12. Hui JI. Outcomes of orbital implants after evisceration and enucleation in patients with endophthalmitis. Curr Opin Ophthalmol 2010 Sep; 21(5) 375-9.

13. Jordan DR. Klapper SR. Controversies in Enucleation Technique and Implant Selection: Whether to Wrap, Attach Muscles and Peg? In: Guthoff R, Katowitz, JA, eds. Oculoplastics and Orbit: Aesthetic and Functional Oculofacial Plastic Problem Solving in the 21st Century, Volume 3. Berlin Heidelberg: Springer, 2010: 195-206.

14. Shields JA, Shields CL, Demirci H, et al. Experience with eyelid-sparing orbital exenteration: the 2000 Tullos O. Coston Lecture. Ophthal Plast Reconstr Surg 2001 Sep ;17(5): 355-61.

15. Tyers AG. Orbital Exenteration for invasive skin tumors. Eye 2006; 1165-1170.

16. Soysal HG. Orbital exenteration: a 10-year experience of a general oncology hospital. Orbit 2010 Jun; 29(3): 135-9.

17. Rahman I, Cook AE, Leatherbarrow B. Orbital exenteration: a 13 year Manchester experience. Brit J Ophthalmol 2005 October; 89(10): 1335-1340.

18. Nassab RS, Thomas SS, Murray D. Orbital exentera- 
tion for advanced periorbital skin cancers: 20 years experience. J Plast Reconstr Aesthet Surg 2007; 60(10):1103-9.

19. Bartley GB, Garrity JA, Waller RR, et al. Orbital exenteration at the Mayo Clinic. Ophthalmology 1989; 96: 468-73.

20. Ramey N, Gupta D, Price K, et al. Comparison of Complication Rates of Porous Anophthalmic Orbital Implants. Ophthalmic Surgery, Lasers and Imaging. Sep 2011: 434-40.

21. Chalasani R, Poole-Warren L, Conway RM, BenNissan B. Porous orbital implants in enucleation: a systematic review. Surv Ophthalmol 2007 Mar-Apr; 52(2): 145-55.

22. Alford M. Enucleation, Evisceration and Exenteration Techniques. Duane's Clinical Ophthalmology CD rom online. Volume 5, Chapter 82. Lippincott, Williams and Wilkins. http://80.36.73.149/almacen/medicina/ oftalmologia/enciclopedias/duane/index.html.

23. www.ocularist.org

24. Raizada K, Rani D. Ocular Prosthesis. Contact Lens and Anterior Eye 2007; 30:152-162.

25. Patil SB, Meshramkar R, Naveen BH, Patil NP. Ocular Prosthesis: A Brief Review and Fabrication of an Ocular Prosthesis for a Geriatric Patient. Gerodontology $2008 ; 25: 57-62$

26. Zuravleff JJ, Hughes MO. Evisceration of the Human Eye with Ocular Prosthetic Restoration. J Ophthal Prosthetics 2011; 16:7-14.

27. Kaltreider, SA. The Ideal Ocular Prosthesis Analysis of Prosthetic Volume. Ophthal Plast Reconstr Surg 2000; 16(5):388-392.
28. Jordan DR. Problems After Evisceration Surgery With Porous Orbital Implants, Experience with 86 Patients. Ophthal Plast Reconstr Surg 2004; 20(5): 374-380.

29. Pine K, Sloan B, Stewart J, Jacobs R. Concerns of Anophthalmic Patients Wearing Artificial Eyes. Clin Exper Ophthalmol 2011; 39:47-52.

30. Han, JW, Yoon, JS, Jang, SY. Short-term effects of topical cyclosporine A $0.05 \%$ (Restasis) in long-standing prosthetic eye wearers: a pilot study. Eye (2014) 28 , 1212-1217; doi:10.1038/eye.2014.174; published online 1 August 2014

31. Tawfik HA, Raslan AO, Talib N. Surgical Management of Acquired Socket Contracture. Current Opin Ophthalmol 2009; 20:406-411.

32. Bajaj MS, Pushker N, Singh KK, Chandra M, Ghose S. Evaluation of Amniotic Membrane Grafting in the Reconstruction of Contracted Socket. Ophthal Plast Reconstr Surg 2006; 22(2): 116-120.

33. Li D, Jie Y, Liu H, Liu J, Zhu Z, Mao C. Reconstruction of Anophthalmic Orbits and Contracted Eye Sockets With Microvascular Radial Forearm Free Flaps. Ophthal Plast Reconstr Surg 2008; 24(2): 94-97.

34. Wei, HY, Liao, SL. The reconstruction of a contracted eye socket using a post-auricular full-thickness skin graft. Graefes Arch Clin Exper Ophthalmol May 2014; 252(5: 821-827.

35. Mombaerts I, Groest E. Upper Eyelid Ptosis Surgery Using a Preparatory Ocular Prosthesis. Ophthal Plast Reconstr Surg 2009; 25(2): 90-93.

36. Fahim, DK, Frueh, BR, Musch, DC, Nelson, CC. Complications of Pegged and Non-pegged Hydroxyapetite Orbital Implants. Ophthal Plast Reconstr Surg 2007; 23(3): 206-210

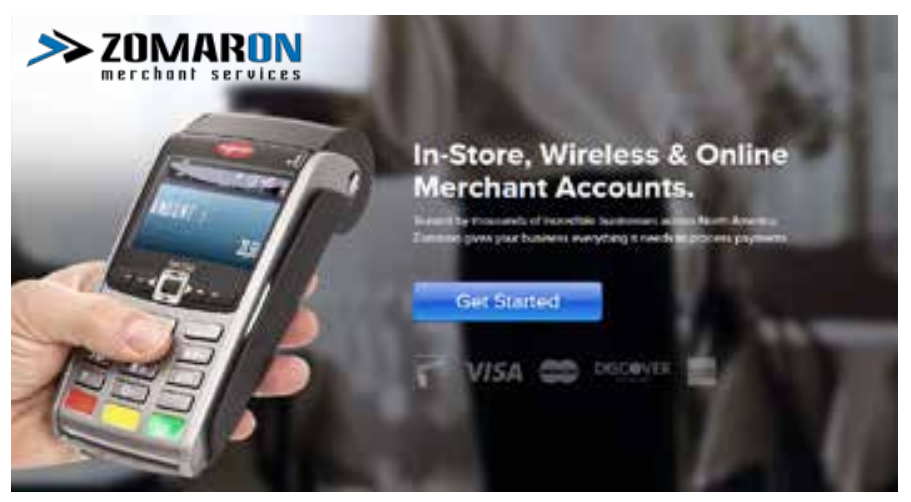

ZOMARON

$\mathbf{S}$

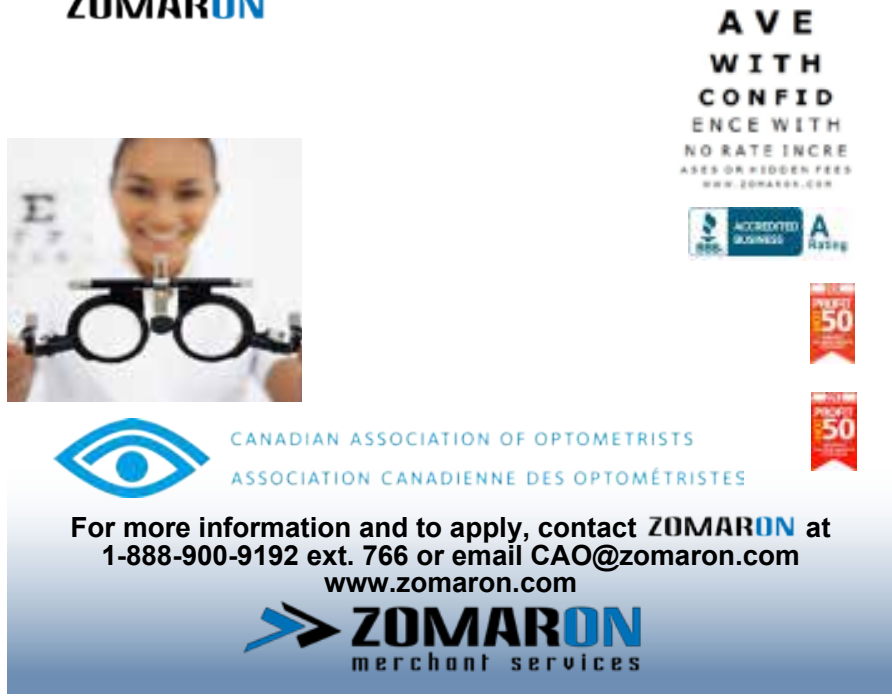

